\title{
Serial position effects in short-term visual memory: A SIMPLE explanation?
}

\author{
Dennis C. HaY and MaRY M. SMyth \\ Lancaster University, Lancaster, England \\ GRAHAM J. HiTCH \\ University of York, York, England \\ AND \\ NeIL J. Horton \\ Lancaster University, Lancaster, England
}

\begin{abstract}
A version of Sternberg's (1966) short-term visual memory recognition paradigm with pictures of unfamiliar faces as stimuli was used in three experiments to assess the applicability of the distinctiveness-based SIMPLE model proposed by Brown, Neath, and Chater (2002). Initial simulations indicated that the amount of recency predicted increased as the parameter measuring the psychological distinctiveness of the stimulus material (c) increased and that the amount of primacy was dependent on the extent of proactive interference from previously presented stimuli. The data from Experiment 1, in which memory lists of four and five faces varying in visual similarity were used, confirmed the predicted extended recency effect. However, changes in visual similarity were not found to produce changes in $c$. In Experiments 2 and 3, the conditions that influence the magnitude of $c$ were explored. These revealed that both the familiarity of the stimulus class before testing and changes in familiarity, due to perceptual learning, influenced distinctiveness, as indexed by the parameter $c$. Overall, the empirical data from all three experiments were well fit by SIMPLE.
\end{abstract}

The vast majority of investigations of serial memory have been conducted within the verbal domain. These have shown familiar bow-shaped serial position functions, using a variety of paradigms, including probed recall (Avons, Wright, \& Pammer, 1994; Nairne, Whiteman, $\&$ Woessner, 1995) and serial reconstruction (Nairne, Riegler, \& Serra, 1991). Similar results arise if the stimulus materials are familiar pictures that can be verbally encoded (e.g., Manning \& Schreier, 1988). The shape of the serial position curve and the error transposition patterns, however, are not a consequence of employing materials capable of being verbally encoded. Recent research has shown that serial reconstruction tasks in which random matrices (e.g., Avons, 1998) and unfamiliar faces accompanied by verbal suppression (Smyth, Hay, Hitch, \& Horton, 2005) are used yield similarly shaped bow-shaped curves and similar transposition error patterns.

In contrast, when memory for visual stimuli is examined using probed recognition, the typical finding is not of a bow-shaped serial position curve but of one with no primacy and only last-item recency. Phillips and Christie (1977) first demonstrated this nonstandard serial position curve, using a range of paradigms, and this finding has been replicated with a variety of materials and methods (e.g.,
Avons, 1980, 1998; Broadbent \& Broadbent, 1981; Hanna \& Loftus, 1993; Kerr, Avons, \& Ward, 1999; Korsnes, Magnussen, \& Reinvang, 1996; Walker, Hitch, \& Duroe, 1993; Ward, Avons, \& Melling, 2005).

Two different forms of models have been proposed to explain the results from tasks investigating the probed recognition of visual stimuli. One is a domain-specific explanation first proposed by Phillips and Christie (1977), which postulates two distinct forms of visual memory representation. The first component is a newly generated internal representation, which Phillips and Christie termed a stable long-term visual memory. The second is a representation held in a fragile short-term visual memory (STVM) store with a capacity limited to a single item. In this model, attention is allocated to each presented item in turn. Each item is maintained by a process of visualization in STVM, and this is used to derive the internal representation. With presentation of the next stimulus, attention is switched to encoding that pattern. Recognition is superior for the pattern being maintained in the limited capacity STVM, thus explaining the superior last-item performance, and is poorer for items held in the long-term store. This model predicts single-item recency for all forms of novel visual material that cannot be verbally encoded. 
An alternative, domain-independent interpretation of the serial position effects observed with visual stimuli was presented by Neath (1993), who invoked the concept of distinctiveness as the explanatory factor. Employing a variant of Murdock's (1960) model, he presented digitized snowflake designs in a probe recognition task, arguing that the insertion of an interval between items in a list makes them temporally distinct. These experiments showed good fits between the performance predicted by the mathematical model of distinctiveness and the observed data. However, in the original Neath model, distinctiveness is based solely on the temporal relationships between items, and other forms of distinctiveness (e.g., the intrinsic distinctiveness of the stimuli) were not taken into account. In a series of experiments designed to examine the applicability of this model, Kerr et al. (1999) presented faces and random matrices in an STVM task and concluded that their results failed to support the predictions made by the dimensional distinctiveness model.

A more recent computational instantiation of temporal distinctiveness is the scale invariant memory, perception and learning (SIMPLE) model proposed by Brown, Neath, and Chater (2002; see also Neath \& Brown, 2006). As applied to STVM tasks, this model distinguishes two forms of distinctiveness. The first is again based on the temporal relationships between items, with temporally distant items being more confusable. The second captures the psychological distance between items, reflecting characteristics such as visual similarity that make items confusable. We will refer to this as psychological distinctiveness. In this model, recall probability is inversely related to confusability, with more recent items viewed as less confusable and, hence, easier to recall (Brown et al., 2002). Specifically, the confusability between any two memory items is related to the time between their encoding and retrieval. The probability of correctly identifying a probe as a memory item (responding $\mathrm{R}_{j}$ given a stimulus $\mathrm{S}_{j}$ ) is given by

$$
\begin{aligned}
P\left(\mathrm{R}_{j} \mid \mathrm{S}_{i}\right)= & \frac{\operatorname{ratio}\left(\mathrm{S}_{i}, \mathrm{~S}_{j}\right)^{c}}{\sum_{k} \operatorname{ratio}\left(\mathrm{S}_{i}, \mathrm{~S}_{k}\right)^{c}}, \\
& \text { where ratio }\left(\mathrm{S}_{i}, \mathrm{~S}_{j}\right)=\frac{\mathrm{S}_{i}}{\mathrm{~S}_{j}} \text { if } \mathrm{S}_{i}<\mathrm{S}_{j}, \\
& \text { and ratio }\left(\mathrm{S}_{i}, \mathrm{~S}_{j}\right)=\frac{\mathrm{S}_{j}}{\mathrm{~S}_{i}} \text { if } \mathrm{S}_{j} \leq \mathrm{S}_{i} .
\end{aligned}
$$

Thus, earlier items interfere with the recognition of later items. However, SIMPLE can be viewed as a local distinctiveness model in which this proactive interference is generated by a small number of previous items. In Brown et al. (2002), the size of the locality producing interference is never specified but is typically implemented by including only items from the same memory list, although they also report simulations of the Brown-Peterson paradigm, in which items from previous trials were the source of proactive interference.

Using the timing parameters commonly found in empirical studies (e.g., Kerr et al., 1999; Neath 1993)—that is, a stimulus presentation time of $1 \mathrm{sec}$, an interitem presentation interval (IPI) of $1 \mathrm{sec}$, and a retention interval (RI) of $3 \mathrm{sec}$ between presentation of the last item and presentation of the probe-it is possible to calculate the response probabilities. For example, the probability of correctly identifying the last item (Item 5) is

$$
P\left(\mathrm{R}_{5} \mid \mathrm{S}_{5}\right)=\frac{\left(\frac{3}{3}\right)^{c}}{\left(\frac{3}{11}\right)^{c}+\left(\frac{3}{9}\right)^{c}+\left(\frac{3}{7}\right)^{c}+\left(\frac{3}{5}\right)^{c}+\left(\frac{3}{3}\right)^{c}}
$$

The power index $c$ in the SIMPLE model takes into account the psychological distance between items. When $c=1$, items are seen as being similar, with the probability of correctly identifying the last item being $P\left(5 \mid \mathrm{R}_{5}\right)=$ .380; for correct retrieval of each of the previous items, the values are $P\left(4 \mid \mathrm{R}_{4}\right)=.301, P\left(3 \mid \mathrm{R}_{3}\right)=.281, P\left(2 \mid \mathrm{R}_{2}\right)=$ .287 , and $P\left(1 \mid \mathrm{R}_{1}\right)=.314$, clearly showing the reductions in the probability of identifying earlier items.

The power index $c$ governs the rate at which confusability decreases as items become more psychologically distinct. When psychologically distinct items are employed (e.g., when $c=5$ ), the probabilities of correctly identifying items in the different temporal positions become $P\left(5 \mid \mathrm{R}_{5}\right)=.911, P\left(4 \mid \mathrm{R}_{4}\right)=.748, P\left(3 \mid \mathrm{R}_{3}\right)=.629$, $P\left(2 \mid \mathrm{R}_{2}\right)=.585$, and $P\left(1 \mid \mathrm{R}_{1}\right)=.670$. This indicates that although overall performance increases, the temporal advantage enjoyed by the most recent items remains. In addition, with higher values of $c$, the amount of primacy and recency increases.

In addition, as the number of items presented increases, the number of components in the function denominator increases, resulting in greater amounts of proactive interference that reduce the probability of correct recognition. Thus, the amount of proactive interference also depends crucially on the size of the interference locality.

Given the key roles played by the $c$ parameter and the size of the interference locality in the SIMPLE model, our first task was to explore how performance is predicted to vary in a simulated STVM probe recognition task.

\section{SIMPLE Simulations of STVM Performance}

Examinations of STVM have generally employed some form of episodic recognition task. Since the most basic form of this task - and one of the easiest to model-is that pioneered by Sternberg (1966), we chose this as the basis of a series of simulations. In this task, a memory list is presented, followed by a probe, and participants then make yes/no judgments on the basis of memory list membership. We simulated performance with memory lists of four or five items, using an IPI of $2 \mathrm{sec}$ (i.e., a 1-sec presentation, followed by a 1-sec gap), an RI of $3 \mathrm{sec}$ before the presentation of the probe (i.e., the standard postitem interval of $1 \mathrm{sec}$ plus an additional $2 \mathrm{sec}$ ), and an intertrial interval (ITI) of $3 \mathrm{sec}$. The ITI was estimated from the Kerr et al.'s (1999) experiments, in which participants had to respond and wait $2 \mathrm{sec}$ before commencement of the next trial. The results of varying the psychological dis- 

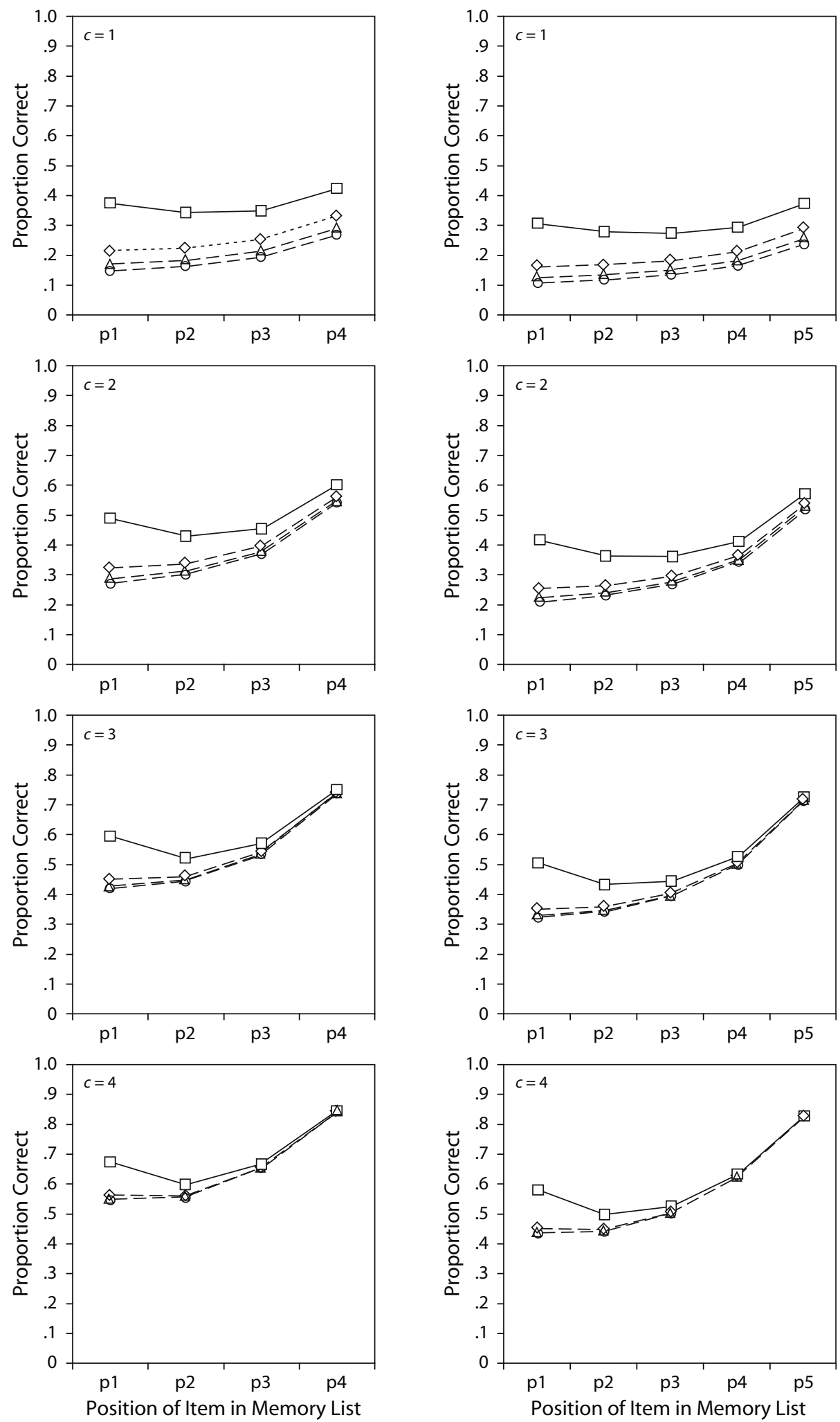

Figure 1. Serial position functions produced by the SIMPLE model from simulations of a visual probe task with an interim presentation interval of $2 \mathrm{sec}$ and a retention interval of $3 \mathrm{sec}$. These simulate performance from a list of four memory items (left panels) and five memory items (right panels) in which $c$ took the values $1,2,3$, and 4 . The upper function in each plot is a result of allowing only items from the current trial to produce interference. The lower functions result from allowing interference from one through three previous trials. 
tinctiveness of the memory and probe items are shown in Figure 1.

This illustrates two effects that are useful for evaluating SIMPLE. (1) The first is an increasing recency gradient. As $c$ increases, so does the magnitude and extent of recency, culminating in a clearly discernible recency gradient when $c$ has a value of four. This is particularly noticeable in the upper functions in each panel, where only the items in a single trial produce interference effects. Such function shapes are clearly at odds with those normally reported, where only single-item recency has been observed. However, singleitem recency has been found in studies in which novel stimuli, such as random matrices (e.g., Avons, 1990,1998; Phillips \& Christie, 1977), wallpaper patterns (Broadbent \& Broadbent, 1981), and snowflakes (Neath, 1993), have been used. It may be that such functions are representative of stimuli that have low psychological distinctiveness, being more similar to the functions generated with low values of $c$, which do tend to show only last-item recency.

(2) The second effect is an increasing primacy effect. Primacy in SIMPLE is an example of an edge effect. Since this is a local distinctiveness model, interference effects accrue from an item's temporal neighbors. Thus, the initial item in a list is relatively temporally distinct from previous trial items and, hence, suffers less proactive interference. As can be seen in the upper functions in each of the panels in Figure 1, primacy increases as the psychological distinctiveness of items increases. Again, this is at odds with the reported function shape when single-item recency is found, since this is flat over the initial serial positions. As with the recency effect, it may be that the low levels of primacy occurring with abstract visual stimuli go undetected. However, there is another possibility. In STVM tasks, the next trial commences almost immediately after a response has been made. Thus, the items from previous trials are temporally near to items in the next trial, making these items likely neighborhood candidates. We examined such influences by systematically increasing the numbers of prior trial items that were allowed to produce proactive interference effects. The lower functions in each panel in Figure 1 are from simulations in which the locality of the interference effects was extended to include items from increasing numbers of previous trial items. When the interference locality was extended to include only the items from the previous trial, this produced noticeable reductions in the magnitude of the primacy effect. We also noted that adding items from increasing numbers of trials produced little additional effect. Thus, our simulations suggest that the usual observed lack of primacy may result from the existence of a larger interference locality.

The rationale behind the series of STVM experiments reported here was twofold: first, to explore the applicability of the SIMPLE model to the domain of memory for a range of visual stimuli and, second, to examine the primacy and recency predictions made by the model.

\section{EXPERIMENT 1}

Previous investigations of STVM have tended to employ abstract stimulus classes, which are likely to be low in psy- chological distinctiveness. These have tended to produce functions with only last-item recency, which resemble the functions predicted by SIMPLE when the value of $c$ is low. However, as $c$ increases, SIMPLE predicts more than lastitem recency. Thus, identifying a stimulus class in which items are more psychologically distinct would allow direct examination of the extent of the recency effect and the predictions made by the SIMPLE model.

Unfamiliar faces are such a stimulus class. Because faces are a highly familiar stimulus class, these are likely to produce more psychologically distinct memory representations than did the abstract visual stimuli previously employed. This view is based on the body of evidence indicating the existence of a specialized system for the rapid encoding and efficient storage of faces (Bruce \& Young, 1986; Hay \& Young, 1982). More important, there is evidence to support the view that faces are represented in a multidimensional face space specifically constructed to encode distinctiveness (Valentine, 1995; Valentine \& Endo, 1992). In this representational space, the center is assumed to represent the average face on each dimension, the dimensions being those that best serve to discriminate exemplars. Thus, this face space model is an example of the class of models that are generalizations of signal detection theory and multidimensional scaling models (e.g., Ashby \& Townsend, 1986; Nosofsky, 1986). One key underlying assumption in this view is that distinctiveness can be judged only relative to the population of previously encountered exemplars (Murdock, 1960). Since faces are highly salient and frequently encountered in everyday life, a highly populated, well-defined representational space already exists. This is in contrast to the abstract stimuli previously employed to investigate STVM that have been infrequently or, more usually, never previously encountered. Thus, faces are a class of stimuli that allows us to investigate SIMPLE under conditions in which the $c$ parameter is considerably higher than it has been in previous investigations.

Interestingly, there already exist hints in the literature that faces may not yield the typical last-item recency effect when used in probed recognition tasks. The basis for this assertion is the data from the set of experiments reported by Kerr et al. (1999), who examined STVM performance using Mac-a-Mug (i.e., schematic) faces and random matrices. They found that matrices only exhibited a last-item recency effect with an RI of $0 \mathrm{sec}$ (Experiment 1B). In contrast, similar presentation conditions with their facial stimuli suggested that the recency effect might extend over the last two items (Experiment 1E). Unfortunately, no firm conclusions can be drawn, since details of the relationships between the relevant serial position effects were not reported.

Another consequence of the face space model is that the representations of visually similar faces will be clustered in neighborhoods and be less distinct than those of visually dissimilar faces. Thus, manipulating the visual similarity of faces within a memory list should produce variations in the distinctiveness of the representations within a single trial. In the first experiment reported, we employed faces as the stimulus class likely to produce more psychologically distinct representations than would abstract patterns. To further manipulate psychological distinctiveness, we also sys- 


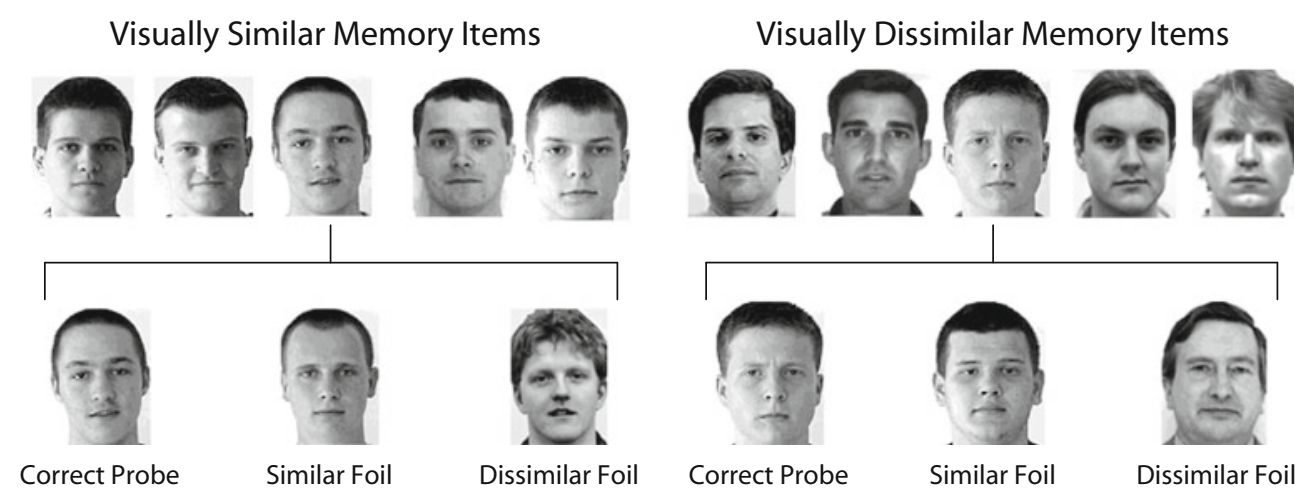

Figure 2. Examples of the visually similar and dissimilar faces used in Experiment 1 and examples of the test items used to examine recognition performance.

tematically varied the visual similarity of unfamiliar faces within a set. In one condition, memory lists contained only visually similar faces, whereas in another, there were only visually dissimilar faces. Hunt (2003) has stressed the relativity of distinctive processing, with this operating not only to identify correct items, but also to reject incorrect items. That is, distinctive processing may depend crucially on the relationship between the memory items and the foils used in such tasks. In addition, Kerr et al. (1999) have already noted inconsistencies in the design of STVM probe experiments, with foils being derived from one particular memory item in some experiments (e.g., Phillips \& Christie, 1977), whereas foils unrelated to the memory items are used in others (e.g., Neath, 1993). Here, we used two forms of foils chosen to be either visually similar to one of the memory set items or visually dissimilar to all the memory faces.

Last, we examined the contribution of verbal encoding in STVM tasks by comparing performance with and without verbal suppression. Although there is a body of evidence that suggests that unfamiliar faces are difficult to encode verbally (e.g., for an early review, see Ellis, 1975; Hay \& Young, 1982), it remains a possibility that any form of additional encoding could enhance item distinctiveness. If this is the case, manipulating the availability of verbal encoding should lead to serial position functions with different characteristics.

\section{Method}

Participants. Forty-eight males and 48 females were recruited from the student population of Lancaster University and were paid $£ 5$ to participate in this experiment. All had normal or corrected-to-normal vision and were fluent in English. Each participant was allocated to one of eight experimental conditions.

Materials. Grayscale images of full frontal poses of Caucasian faces were selected from various public domain databases and the Lancaster University Psychology Department's face library. These were cropped to minimize the background and maximize the size of a face before being set to a standard height of $37.5 \mathrm{~mm}$ when displayed on a computer monitor.

Faces were selected to form 112 sets of six faces. One hundred eight sets were used for the experimental stimuli, with half the sets being composed only of male faces and half only of female faces. The remaining 4 sets were used for practice stimuli. In addition, sets were consistent in terms of the age of the faces (either 18-30 or
45-65 years), hair color (dark or light), hair length (short or long), and, on the basis of judgment of the experimenters, visually similar facial features. The resulting sets provided the memory items, the probes (an example of one of the memory items), and the foils (a stimulus face that was not one of the memory items) for the individual experimental trials. The faces were grouped for use in four different conditions. The conditions differed in the composition of the memory items and the relationship of the foil items to the memory items. Two of the conditions had memory items drawn from a single list (similar items), and two had the individual items drawn at random without replacement from all of the faces of the same gender (dissimilar items). For conditions requiring similar foils, an item was selected randomly from the items in a set not used as in the memory list. In the conditions requiring dissimilar foils, these were drawn randomly from all the items of the same gender not used as memory items. Examples can be found in Figure 2.

The faces were displayed against a white background on an Apple iMac computer running the SuperLab application and were positioned at approximately eye level at a distance of $75 \mathrm{~cm}$ from the participants. The display resolution was set to $1,024 \times 768$ pixels, with a refresh rate of $75 \mathrm{~Hz}$. The participants responded by pressing one of two keys on the computer keyboard.

Procedure. The participants were tested individually and were informed that their memory for faces was to be examined. This would involve the presentation of a list of four or five unfamiliar faces, followed by a test face. Their task was to decide whether this was or was not one of the items from the previously presented list. The Q and the ] keys on the computer keyboard were used to record performance, with the relationship between the keys and yes/no decisions being counterbalanced over participants. Four practice trials were conducted before the presentation of two experimental blocks. List length four always preceded list length five. The order of trials within blocks was randomized for each participant.

Each trial started with the central presentation of the word "ready" for $1,000 \mathrm{msec}$, which acted both as an indication of the start of a trial and as an instruction to begin the concurrent vocal suppression task. After $500 \mathrm{msec}$, this was replaced by the list of memory faces. Each face was presented for $1,000 \mathrm{msec}$ and was separated from the subsequent face by a 1,000-msec blank screen. Each face was presented $37.5 \mathrm{~mm}$ above the center of the display. Following the last item, there was a 3,000-msec gap, during which the screen was blank before the presentation of the test face. The probe was presented $1.5 \mathrm{in}$. below the center of the display. The participants were instructed to press one of the keys if the test face was a member of the memory list or to press the other key to indicate nonmembership and to be as accurate and as fast as possible when making a decision. Once a response had been made (or a 5-sec time limit had elapsed), the probe face disappeared, and the next trial began after a 1,000-msec delay. The participants were given a short break between trial blocks. No feedback was supplied concerning 
Table 1

Performance Indices Averaged Over List Position for Each Memory List Length Used in Experiment 1

\begin{tabular}{|c|c|c|c|c|c|c|c|c|c|}
\hline \multirow[b]{2}{*}{ List Type } & \multirow[b]{2}{*}{ Foil Type } & \multicolumn{4}{|c|}{ List Length 4} & \multicolumn{4}{|c|}{ List Length 5} \\
\hline & & $p$ (hit) & $A^{\prime}$ & $B_{\mathrm{D}}^{\prime \prime}$ & $c$ & $p$ (hit) & $A^{\prime}$ & $B_{\mathrm{D}}^{\prime \prime}$ & $c$ \\
\hline Similar & similar & .77 & .88 & .56 & 5.77 & .74 & .86 & .25 & 5.82 \\
\hline Similar & dissimilar & .80 & .92 & -.01 & 6.09 & .77 & .89 & .34 & 6.20 \\
\hline Dissimilar & similar & .80 & .88 & .12 & 6.11 & .74 & .87 & .30 & 6.67 \\
\hline Dissimilar & dissimilar & .83 & .89 & .37 & 6.85 & .70 & .86 & .48 & 6.08 \\
\hline Mean faces & & .80 & .89 & .26 & 6.21 & .74 & .87 & .34 & 6.19 \\
\hline
\end{tabular}

Note $-p$ (hit) is the proportion of correctly identified probes, $A^{\prime}$ and $B_{\mathrm{D}}^{\prime \prime}$ are the nonparametric signal detection parameters estimating discriminability and response bias. $c$ refers to the mean value of the power parameter from the best-fitting SIMPLE function for each participant.

the accuracy of the responses given, and the participants were unable to amend their response once this had been made.

Design. Each participant was allocated at random to one of the two main experimental conditions. In the first, the participants were informed that they would be required to repeat the numbers $1,2,3,4$ when the ready signal appeared and to continue vocalizing until they had made a response. Each participant then practiced this concurrent task until the experimenter was satisfied that both the repetition rate and the volume of their speech were appropriate. In the second condition, the participants performed without a concurrent task. Within each of the two conditions, the participants were further allocated to one of four other experimental conditions in which both memory items and foils were either visually similar or dissimilar.

Each participant completed a block of 48 trials with four memory items per list, with equal numbers of probe and foil trials, and then a block of 60 trials with five memory items from the same experimental condition. In both blocks, there were equal numbers of male and female memory lists. On probe trials, each memory item position was probed six times, with the order of position probed being randomized for each participant.

This yielded a $2 \times 2 \times 2 \times 4 \times 2$ mixed design (presence of verbal suppression $\times$ similarity of the memory items $\times$ similarity of the foil items $\times$ probe position $\times$ memory list size), in which the first three factors were between factors and the last two within factors.

\section{Results}

Performance analyses. Since the paradigm used in this study does not allow false alarms or correct rejections to be explicitly related to memory list position, data for the initial analyses calculated indices collapsed over serial position. The proportion of hits for each participant was calculated over list position and subjected to a $2 \times 2 \times$ $2 \times 2$ mixed factor ANOVA (presence of verbal suppression $\times$ similarity of the memory items $\times$ similarity of the foil items $\times$ memory set size). The only effect found to be reliable was the manipulation of list length $[F(1,88)=$ $26.70, M S_{\mathrm{e}}=0.0063, p<.0001, \eta^{2}=.23$ ], with recognition being easier with four items than with five items (see Table 1). Interestingly, the mean recognition difference from simulations in which $c$ varied between 1 and 6 was found to be .064, with a standard deviation of .004, which agrees well with the observed difference of .062. The proportion of correct rejections over memory list positions in each of the experimental conditions was also calculated and similarly analyzed. The results indicated that employing verbal suppression did not influence performance, nor did this interact with any other factor. An effect of probe similarity was observed $\left[F(1,88)=16.76, M S_{\mathrm{e}}=\right.$
0.0095, $p<.0001, \eta^{2}=.16$ ], indicating better rejection of visually dissimilar foils than of visually similar foils (see Table 1).

Nonparametric signal detection indices $A^{\prime}$ and $B_{\mathrm{D}}^{\prime \prime}$ (Pollack \& Norman, 1964), measuring discriminability and response bias, respectively, were also calculated from the recognition data. These are also shown in Table 1. A $2 \times 2 \times 2 \times 2$ mixed factor ANOVA (presence of verbal suppression $\times$ similarity of the memory items $\times$ similarity of the foil items $\times$ memory set size) of the $A^{\prime}$ scores revealed that items in memory sets with four faces were more discriminable than items in memory set of five faces $\left[F(1,88)=12.16, M S_{\mathrm{e}}=0.0021, p<.01, \eta^{2}=.12\right]$. In addition, the manipulation of foil similarity was found to be reliable $\left[F(1,88)=4.04, M S_{\mathrm{e}}=0.0043, p<.05, \eta^{2}=\right.$ .05 ], with discriminability better with visually similar than with visually dissimilar foils. However, this effect was moderated by the interaction between foil similarity and the similarity of the memory items $[F(1,88)=3.98$, $\left.M S_{\mathrm{e}}=0.0021, p<.05, \eta^{2}=.05\right]$. Additional analyses using the Tukey test ( $\alpha=.05$ ) indicated better discrimination when visually similar memory set faces were tested with visually dissimilar foils. A similar analysis of the response bias indices revealed one reliable effect associated with foil similarity $\left[F(1,88)=3.98, M S_{\mathrm{e}}=0.0021\right.$, $p<.001, \eta^{2}=.17$ ], with visually similar foils producing more conservative responding than did visually dissimilar probes.

Serial position effects were examined by calculating the mean proportion of hits for each of the serial positions. The data from the trials having four memory faces were subjected to a $2 \times 2 \times 2 \times 4$ mixed design ANOVA (presence of verbal suppression $\times$ similarity of the memory items $\times$ similarity of the foil items $\times$ probe position). No main effects associated with verbal suppression, memory list similarity, or foil similarity and no interactions involving these factors were observed. However, a reliable main effect of serial position $\left[F(3,264)=44.12, M S_{\mathrm{e}}=0.0026\right.$, $p<.001, \eta^{2}=.34$ ] was found (see Figure 3). To examine the magnitude of the recency advantage, comparisons between the means of successive serial positions were conducted using Bonferroni-adjusted multiple comparisons. These indicated reliably better performance for Position 4 when compared with Position 3, significantly better performance for Position 3 when compared with Position 2 and no performance difference between Positions 1 and 2. 
A similar analysis strategy was applied to the trials having five memory items. As before, no reliable effects involving verbal suppression, similarity of the memory list items, or similarity of the foils were found. Again, a reliable effect of serial position was observed $\left[F(4,352)=55.96, M S_{\mathrm{e}}=0.0032, p<.001, \eta^{2}=.34\right]$. The Bonferroni-adjusted tests in this case indicated reliably better performance to probes presented in Position 5 than to those in Position 4, better performance for Position 4 than Position 3, better performance for Position 3 than for Position 2, and no reliable performance difference between Positions 1 and 2 (see Figure 3).

Model fitting. The version of SIMPLE outlined earlier was used to obtain best-fit functions. Initially, the proportion of correct responses at each list position for both list sizes was calculated for each participant, and the resulting data were used to obtain the value of $c$ associated with the best-fitting SIMPLE predictions (see Table 1). The timing parameters used in the simulations were the same as those used in the experiment - that is, an IPI of $2 \mathrm{sec}$, an RI of $3 \mathrm{sec}$, and an ITI of $3 \mathrm{sec}$. The latter value was selected as being a reasonable estimate, since the mean response time observed was approximately $1 \mathrm{sec}$, and the blank screen and the ready signal shown between trials were presented for $1 \mathrm{sec}$ each. The only free parameter in this model was the value of the power index $c$. This was decreased iteratively until the value of root-mean square error (RMSE) between the observed and the predicted data reached a minimum value. The resulting $c$ values obtained from the participants were subjected to a $2 \times 2 \times 2 \times 2$ mixed factor ANOVA (suppression $\times$ similarity of the memory list items $\times$ similarity of the probe $\times$ list size). This revealed no reliable main effects or interactions. That is, neither presence of suppression, change in memory list length, nor any manipulation of visual similarity was found to influence the magnitude of the $c$ parameters associated with faces.

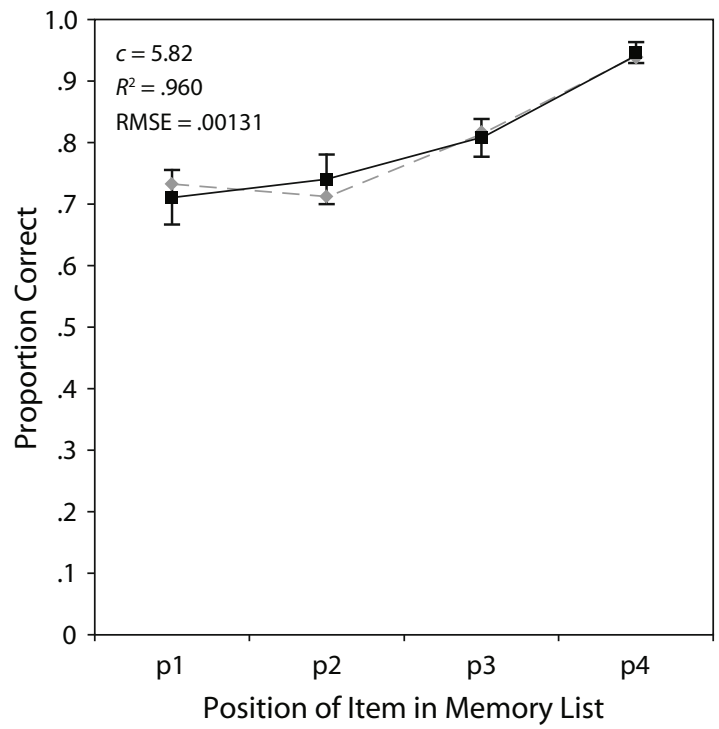

As a result of the failure to find reliable effects from the hits, $A^{\prime}$, and $c$ measures, the proportional hit data at each list position were averaged over the conditions manipulating verbal suppression, memory set similarity, and foil similarity conditions and over participants. Since list length was found not to influence performance, simulations were then run constraining the value of $c$ to be constant across the two list lengths. The prediction that the size of the primacy effect was related to the number of items producing proactive interference was examined by including different numbers of interfering items. This was varied by including only same-list items, by including same-list items and those from one previous list, or including samelist items and those from two previous lists. It was decided to estimate best fit by monitoring the proportional change in RMSE. Best fits were identified as those in which the change values reached or neared a minimum. This was defined either by a reversal of the sign of the value of the proportional change or by the change being less than .05 .

In the case of memory lists with both four and five items, the inclusion of items from the current trial and one preceding trial produced the best-fit SIMPLE functions, which had very similar values of $c$ (see Table 2). Since the ANOVAs had indicated that $c$ did not vary with memory set size, the resulting values of $c$ demarcated the range of values within which to search for the single $c$ value that produced the overall best-fitting functions shown in Figure 3.

\section{Discussion}

The serial position data from Experiment 1 were well fit by the version of SIMPLE employed here (see Figure 3). In addition, the results confirmed that employing faces in a standard STVM probe experiment yielded functions with more than last-item recency. The prediction made by SIMPLE that the shape of the STVM serial position function changes when a more psychologically distinct stimuli class is

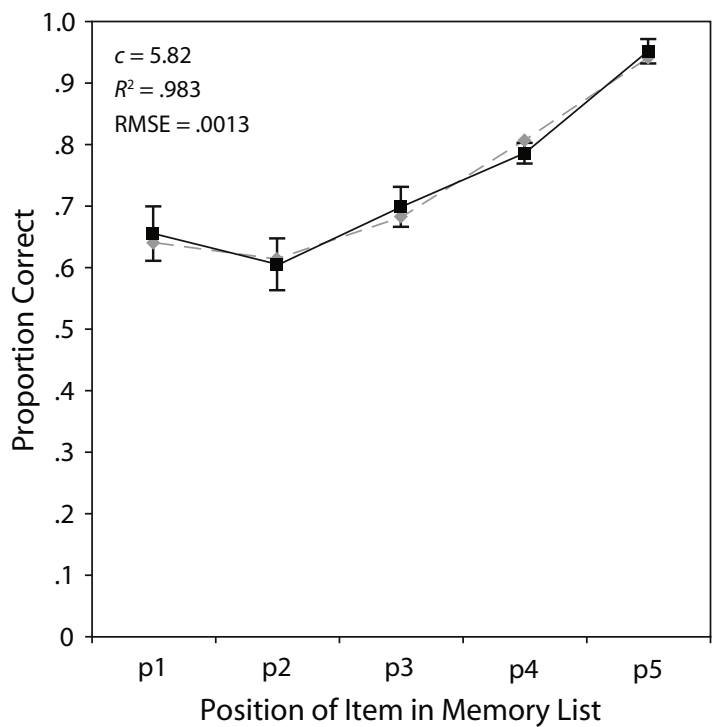

Figure 3. Proportion of faces correctly identified as being previously seen as a function of similarity of the memory set items, similarity of the probe, and serial position in Experiment 1. Data from the trials on which four memory items were presented are shown in the left panel, and those from trials having five memory items are shown in the right panel. 
employed was therefore upheld. Although the informationprocessing model that is frequently offered as an alternative (e.g., Kerr et al., 1999; Phillips \& Christie, 1977) could be modified to explain both the extended recency effect and the lower performance with increasing list length, neither is predicted by the current version. Furthermore, the serial position data from this task, in which successive trials are temporally near, are better fit by SIMPLE functions in which proactive interference arises from a combination of the current list items and the items from the previous trial. This is illustrated by both increased values of the goodnessof-fit index and significantly reduced measures of error, as shown in Table 2. Again, this is consistent with the simulations shown in Figure 1, in which the primacy effect was reduced by increasing the interference locality.

The data also indicated that verbal suppression did not influence performance, supporting the view that novel faces are difficult to verbally encode and negating any possibility of the serial position effects observed having a verbal basis. In contrast, our manipulations of visual similarity were found to influence the discriminability of faces. As was suggested by Hunt (2003), the relationship between the visual characteristics of the foils and the memory items is most important. However, although visual similarity produced changes in overall performance, these did not generate serial position functions with differing $c$ values. It appears that our suggestion that manipulating visual similarity produces changes in the psychological distinctiveness of items is incorrect. Although our manipulations of visual similarity influenced stimulus discriminability, they failed to produce large enough changes in psychological distinctiveness to generate divergent serial position functions. We examined this issue in Experiment 2.

\section{EXPERIMENT 2}

Our original argument was that if the temporal relationships in an STVM task are kept constant, changes in the psychological distinctiveness of the items can be produced by changes in visual similarity, which will, in turn, produce serial position functions with different shapes. In Experiment 1 , we observed serial position functions with an extended recency advantage, with these being equivalent across changes in visual similarity. However, we failed to produce any variation in the $c$ function parameter. This suggests that varying the visual similarity of faces does not produce measurable changes in psychological distinctiveness.

In contrast, differently shaped functions exhibiting only last-item recency have typically been observed with various forms of novel visual stimuli that are difficult to verbally encode. These include random matrices (Kerr et al., 1999; Phillips \& Christie, 1977), block patterns (Korsnes et al., 1996; Walker et al., 1993), snowflake patterns (Neath, 1993), and wallpaper patterns (Broadbent $\&$ Broadbent, 1981). Thus, we attempted to lower psychological distinctiveness and, hence, the value of the $c$ parameter by employing more unfamiliar forms of $a b-$ stract stimuli. Moreover, we used the same temporal relationships between lists and list items as those used in the previous experiment, allowing direct comparisons to be drawn between stimulus types. If the extended recency functions observed in Experiment 1 depended only on the task demands and the temporal relationships between the presented items, similarly shaped functions would obtain. However, if, as the SIMPLE model suggests, function shape is dependent on both temporal and psychological distinctiveness and abstract stimuli differ sufficiently in psychological distinctiveness, more abstract visual stimuli should generate differently shaped functions.

Two different forms of low visual similarity stimuli were generated. The first consisted of examples of the $4 \times 4$ random square matrices initially used by Phillips and Christie (1977) and later by Kerr et al. (1999). The second was constructed by inverting a subset of the faces used in Experiment 1. Inverted faces have the same level of visual complexity as upright faces but do not engage the normal

Table 2

Values of the Power Index $c$ From the Best-Fitting Serial Position Functions for Four and Five Memory Items Generated by Fitting the SIMPLE Model to the Data From Experiment 1 (Faces) and Experiment 2 (Inverted Faces and Random Matrices)

\begin{tabular}{|c|c|c|c|c|c|c|c|c|c|c|}
\hline \multirow[b]{2}{*}{ Stimulus } & \multicolumn{5}{|c|}{ Memory List Length 4} & \multicolumn{5}{|c|}{ Memory List Length 5} \\
\hline & $\begin{array}{l}\text { No. } \\
\text { Items }\end{array}$ & $c$ & $R^{2}$ & RMSE & $\begin{array}{l}\text { Prop. } \\
\text { Change }\end{array}$ & $\begin{array}{l}\text { No. } \\
\text { Items }\end{array}$ & $c$ & $R^{2}$ & RMSE & $\begin{array}{l}\text { Prop. } \\
\text { Change }\end{array}$ \\
\hline \multirow[t]{4}{*}{ Faces } & 4 & 5.52 & .836 & .00545 & & 5 & 5.48 & .955 & .00361 & \\
\hline & 8 & 5.81 & .961 & .00131 & .760 & 10 & 5.84 & .983 & .00127 & .648 \\
\hline & 12 & 5.82 & .963 & .00125 & .046 & 15 & 5.85 & .982 & .00133 & -.048 \\
\hline & 16 & 5.82 & .963 & .00125 & .000 & 20 & 5.85 & .982 & .00134 & -.008 \\
\hline \multirow[t]{4}{*}{ Inverted faces } & 4 & 4.31 & .745 & .00868 & & 5 & 5.25 & .947 & .00598 & \\
\hline & 8 & 4.73 & .906 & .00516 & .406 & 10 & 5.67 & .959 & .00383 & .360 \\
\hline & 12 & 4.76 & .909 & .00526 & -.019 & 15 & 5.69 & .957 & .00606 & -.582 \\
\hline & 16 & 4.77 & .909 & .00527 & -.002 & 20 & 5.69 & .957 & .00609 & -.005 \\
\hline \multirow[t]{4}{*}{ Random matrices } & 4 & 3.44 & .811 & .01150 & & 5 & 3.73 & .917 & .00850 & \\
\hline & 8 & 3.89 & .977 & .00128 & .889 & 10 & 4.18 & .916 & .00754 & .113 \\
\hline & 12 & 3.93 & .978 & .00124 & .031 & 15 & 4.22 & .909 & .00948 & -.257 \\
\hline & 16 & 3.94 & .978 & .00125 & -.008 & 20 & 4.23 & .908 & .00971 & -.024 \\
\hline
\end{tabular}

Note-For each function, two indices of fit between observed and predicted data are presented: the squared correlation $\left(R^{2}\right)$ and the root-mean square error (RMSE). For each stimulus type, fits are provided for differing numbers of items allowed to produce interference effects, along with the proportional change in RMSE associated with each increase. 
Table 3

Performance Indices Averaged Over List Position for Each Memory List Length Used in Experiments 2 and 3

\begin{tabular}{|c|c|c|c|c|c|c|c|c|}
\hline \multirow[b]{2}{*}{ Stimulus Type } & \multicolumn{4}{|c|}{ List Length 4} & \multicolumn{4}{|c|}{ List Length 5} \\
\hline & $p$ (hit) & $A^{\prime}$ & $B_{\mathrm{D}}^{\prime \prime}$ & $c$ & $p$ (hit) & $A^{\prime}$ & $B_{\mathrm{D}}^{\prime \prime}$ & $c$ \\
\hline \multicolumn{9}{|l|}{ Experiment 2} \\
\hline Dissimilar inverted faces & .74 & .77 & .09 & 4.79 & .72 & .75 & .14 & 5.37 \\
\hline Similar inverted faces & .70 & .78 & .21 & 4.55 & .72 & .78 & .00 & 5.06 \\
\hline Random matrices & .64 & .65 & .11 & 3.62 & .62 & .67 & .14 & 4.28 \\
\hline \multicolumn{9}{|l|}{ Experiment 3} \\
\hline Dissimilar inverted faces & .71 & .81 & .17 & 4.63 & .70 & .79 & .02 & 4.72 \\
\hline Similar inverted faces & .68 & .79 & .01 & 4.39 & .63 & .76 & .19 & 4.25 \\
\hline Random matrices & .72 & .80 & .11 & 4.59 & .61 & .76 & .12 & 4.04 \\
\hline
\end{tabular}

Note $-p$ (hit) is the proportion of correctly identified probes, $A^{\prime}$ and $B_{\mathrm{D}}^{\prime \prime}$ are the nonparametric signal detection parameters estimating discriminability and response bias. $c$ refers to the mean value of the power parameter from the best-fitting SIMPLE function for each participant.

face-processing system. The studies of Moses, Ullman, and Edelman (1996), Murray (2004), Murray, Yong, and Rhodes (2000), and Leder, Candrian, Huber, and Bruce (2001) revealed that face inversion destroys the ability to extract expression and identity information by reducing the possibility of employing the forms of configurational processing that are essential for fast and accurate face perception.

However, one of the major differences between matrices and inverted faces is that the latter retain symmetry and consistent feature structure even when inverted. Since this may make them easier to encode than matrices, it was decided to again systematically vary visual similarity by employing the visually similar and the visually dissimilar faces in Experiment 1. This allowed us to examine variations in visual similarity both within and across stimulus classes. In addition, since random matrix foils were derived from one of the list items, only the foils visually related to face list items were used, in an attempt to produce tasks with equivalent demands.

Finally, the possibility that verbal encoding enhances distinctiveness was again investigated. It may be that the fast, efficient encoding mechanisms that exist for faces minimize any involvement of additional encoding mechanisms. However, novel exemplars from unfamiliar visual classes may require a variety of encoding mechanisms to produce maximally distinct memory representations. If such encoding strategies are involved, engaging in verbal suppression should both lower overall performance and change the shape of the serial position function.

\section{Method}

Participants. Thirty males and 42 females were recruited from the student population of Lancaster University and were paid $£ 5$ to participate in this experiment. All had normal or corrected-to-normal vision and were fluent in English.

Materials. Three types of stimuli were used. Two were selected from those used in Experiment 1 and consisted of the faces used in the conditions with visually dissimilar memory items and visually similar probe items and with visually similar memory items and visually similar probe items. These were inverted to produce the experimental stimuli. Exemplars of the third stimulus type were constructed using the procedure described by Phillips and Christie (1977). Briefly, each stimulus was a $4 \times 4$ square matrix having half the squares randomly colored black and the remaining cells white. Each stimulus measured approximately $37.5 \mathrm{~mm}^{2}$. Foils were constructed from one of the memory set items by randomly changing the color of one of the cells.
Procedure. Each participant was tested individually, completing 48 trials with four-item memory lists and 60 trials with five-item memory lists. On half the trials, the probe matched one of the memory items, and on half, it was a foil. The timings and methods of presenting were the same as those employed in the first experiment.

Design. Each participant was allocated at random to one of six experimental conditions. In the first three, the participants completed the recognition task while undergoing the same form of verbal suppression as that employed in the previous experiment. In the remaining three conditions, there was no concurrent task. In these three experimental subconditions, one of three different types of stimulus was used: visually dissimilar inverted faces, visually similar inverted faces, or random matrices. Memory sets contained either four or five items. Together, these yielded a $2 \times 3 \times 2$ design (presence of suppression $\times$ stimulus type $\times$ memory set size).

\section{Results}

Performance analyses. The analysis strategy was similar to that employed in Experiment 1. That is, the data were summarized over list position to produce four performance indices: mean proportion of hits, mean proportion of correct rejections, and the associated signal detection parameters of $A^{\prime}$ and $B_{\mathrm{D}}^{\prime \prime}$. These were individually analyzed using $2 \times 3 \times 2$ mixed factor ANOVAs (presence of verbal suppression $\times$ stimulus type $\times$ list length). Analyses of the hits revealed only one reliable effect, that of stimulus type $\left[F(2,66)=7.89, M S_{\mathrm{e}}=0.0184, p<.001\right.$, $\left.\eta^{2}=.19\right]$. Additional Bonferroni-adjusted comparisons revealed this was due to matrices producing lower recognition levels than did either visually dissimilar or visually similar inverted faces, which did not differ (see Table 3 ).

Consistent with these findings, the analysis of the $A^{\prime}$ values revealed only an effect of stimulus type $[F(2,66)=$ $15.12, M S_{\mathrm{e}}=0.011, p<.001, \eta^{2}=.31$ ], with random matrices being harder to discriminate than either type of inverted faces, which did not differ. Last, no effects related to response bias were observed in this experiment.

As before, the effects associated with serial position were examined separately for each list length. For lists with four items, the proportion of hits for each position was calculated for each participant and was analyzed using a $2 \times 3 \times 2$ mixed ANOVA (presence of suppression $\times$ stimulus type $\times$ list position). A reliable effect of item position was observed $\left[F(3,198)=27.28, M S_{\mathrm{e}}=0.003, p<.0001, \eta^{2}=\right.$ .29]. As in Experiment 1, the magnitude of the recency effect was investigated by conducting additional Bonferroni- 

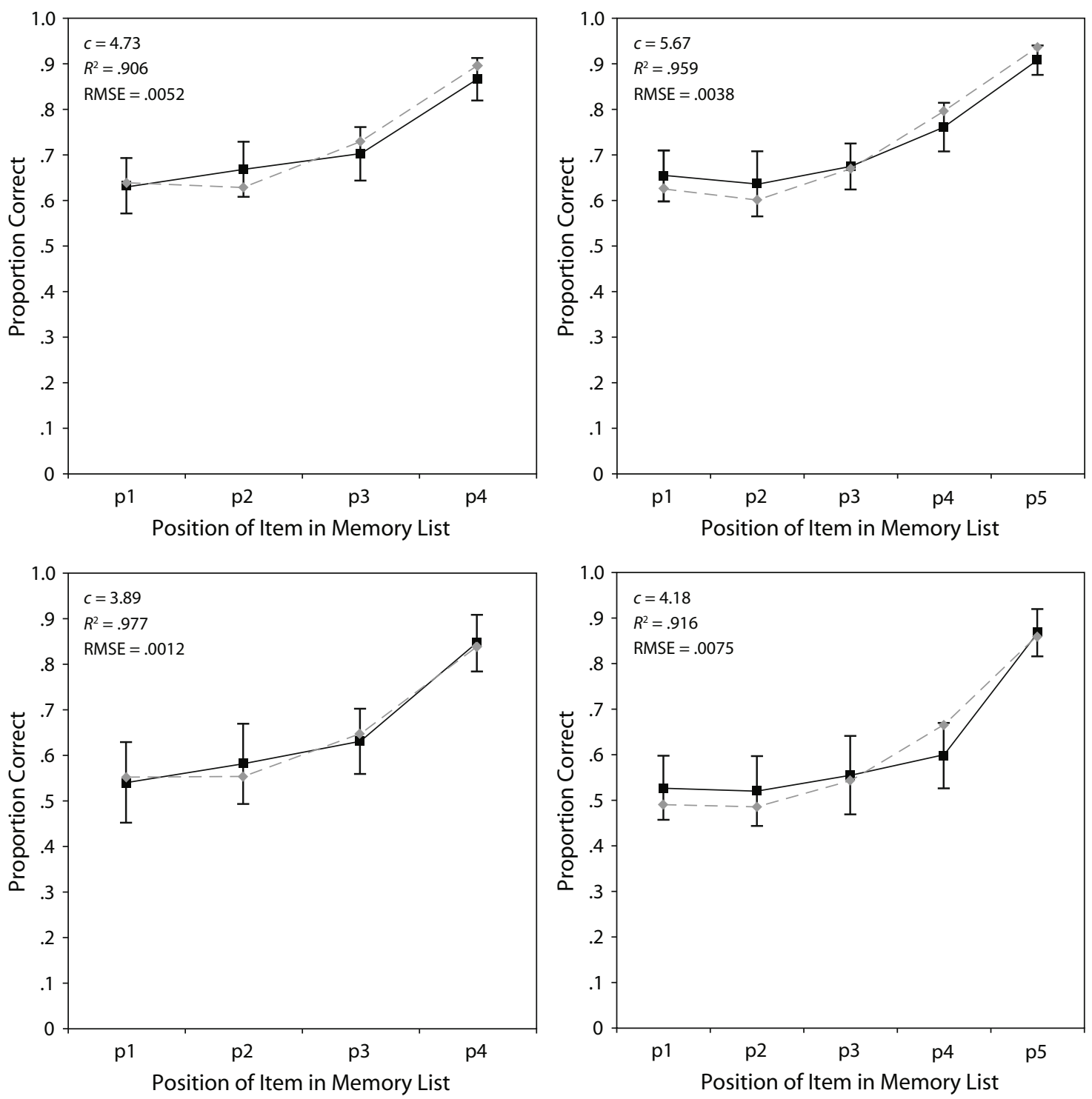

Figure 4. The proportion of inverted faces (upper panels) and matrices (lower panels) correctly identified as being previously seen, as a function of serial position in Experiment 2. Data when four memory items were presented are shown in the left panel, and those when five memory items were presented are shown in the right panel. In this experiment, memory lists with four items always preceded lists with five items.

adjusted comparisons. These revealed that items in Position 4 were better recognized than were those in all the other positions, which did not differ (see Figure 4).

A similar analysis on the data from lists with five memory items also revealed a reliable position effect $\left[F(4,264)=34.89, M S_{\mathrm{e}}=0.0031, p<.001, \eta^{2}=.35\right]$, with Bonferroni-adjusted comparisons indicating items in Position 5 to be better recognized than those in all other positions. It is worth noting that although performance in the initial four positions in inverted faces lists was found to be equivalent, some evidence of an extended recency was observed, with items in Position 4 being better recognized than items in Position 2.

Model fitting. The version of SIMPLE and the timings used in Experiment 1, were again employed to obtain model fits. As before, the proportion of correct responses at each list position for both list sizes was calculated for each participant, and the resulting data were used to obtain the value of $c$ associated with the best-fitting SIMPLE function. The resulting $c$ parameters were subjected to a $2 \times 3 \times 2$ mixed ANOVA (presence of verbal suppression $\times$ stimulus type $\times$ set size), which revealed that $c$ varied with the stimulus type $\left[F(2,66)=13.97, M S_{\mathrm{e}}=1.19, p<.001, \eta^{2}=.30\right]$, with matrices having reliably smaller $c$ values than did either visually similar inverted faces or visually dissimilar inverted faces, which did not differ (see Table 3). In addition, $c$ was observed to vary with list length $\left[F(1,66)=19.44, M S_{\mathrm{e}}=\right.$ $0.83, p<.001, \eta^{2}=.23$ ]. Paradoxically, smaller values of $c$ were found to be associated with memory lists of four items, rather than with lists of five items. 
Since suppression was again found not to influence performance, the hit data were collapsed over this factor. In line with the results from the performance analyses and those involving the $c$ parameter, which consistently indicated no differences between the types of inverted faces, the data were also collapsed over these two forms of inverted faces (see Figure 4). Simulations were then run to fit predictions from SIMPLE to the data for each stimulus type at each of the memory list sizes. As before, the number of items contributing proactive interference was estimated by monitoring the proportional change in RMSE, with best fits being identified using the same criteria as those in Experiment 1.

Consistent with the previous findings, the best-fit functions for both inverted faces and matrices for list lengths of four and five were obtained when items from the current memory list and one preceding list were allowed to produce interference effects (see Table 2).

\section{Discussion}

Taken together, the results from Experiments 1 and 2 confirm the predictions made by SIMPLE, linking the value of the power index to the shape of the serial position curve. They also suggest that the commonly reported flat serial position function with last-item recency is the result of the familiarity of the stimulus materials. In this experiment, the lower familiarity of both inverted faces and matrices, in comparison with faces, was sufficient to produce both lower values of $c$ and the changes in function shape predicted by SIMPLE. At both set sizes, the functions produced were generally flat across all positions save the last. Some evidence of a more extended recency effect was seen with lists of five inverted faces, with items in Position 2 more poorly recognized than those in Position 4 (see Figure 4). As before, the data were well fit by SIMPLE and best fit by the version in which the locality of the interference effects included items from the previous trial. This supports the suggestion that the temporal proximity of the trials in STVM tasks are the cause of the minimal primacy effects typically observed. In addition, the results from this experiment confirmed that verbal encoding does not play a major role in STVM experiments of this kind. Engaging in verbal suppression neither lowered performance nor produced changes in serial position function shape.

However, the one aspect of these data that poses a serious challenge to the SIMPLE model was the reliable change in $c$ related to memory list length. Paradoxically, we found longer memory lists to have higher $c$ values and, hence, to be more psychologically distinct. One interpretation for this pattern of results is that psychological distinctiveness may vary within the course of the experiment. Since unfamiliar stimulus classes are unlikely to have been previously encountered, strategies for the efficient encoding of exemplars and representational structures designed to store exemplars will not exist. As the number of exemplars encountered increases, the more efficient such encoding and representational structures become. In the first two experiments, the block of four-item trials was always presented before the block of five-item trials. Although this may have had minimal impact with highly familiar stimuli, such as faces, it could be that the increased distinctiveness of the items in a list with a length five observed in Experiment 2 was a consequence of some form of perceptual learning. We explored this further in Experiment 3.

\section{EXPERIMENT 3}

The results from Experiments 1 and 2 indicate that changes in the $c$ parameter are associated with changes in stimulus familiarity, with faces being more psychological distinct than inverted faces or matrices. In addition, changes in $c$ were also found to be related to changes in list length, but only for inverted faces and matrices. The latter result could be the result of perceptual learning. In the first two experiments, the participants always completed the four-item memory list trial block first. It may be that for the unfamiliar stimuli, this provided exposure to sufficient numbers of exemplars to allow more efficient encoding and representational structures to develop, thus increasing the distinctiveness of five-item memory list items. If this is the case, reversing the order in which the memory list lengths are encountered should reverse the relationship between $c$ values and list length.

\section{Method}

Participants. Seventeen males and 19 females were recruited from the student population of Lancaster University and were paid $£ 5$ to participate in this experiment. All had normal or corrected-to-normal vision and were fluent in English.

Materials. The inverted faces and the matrix stimuli constructed for the previous experiment were again employed.

Procedure. In all respects save one, the procedure employed in this experiment was identical to that in Experiment 1 . The only change was in the order of presentation, with the trial block of memory lists with five items always preceding the trial block with four memory items for all the participants.

Design. The participants were randomly assigned to one of three conditions: viewing visually similar inverted faces, visually dissimilar inverted faces, or random matrices. This yielded a $3 \times 2$ design (stimulus type $\times$ memory set size). Since verbal suppression was found in the previous experiments not to influence performance, this was not employed here.

\section{Results}

Performance analyses. As in the previous experiments, the data were summarized over list position and four performance indices: Mean proportion of hits, mean proportion of correct rejections, and the associated signal detection parameters of $A^{\prime}$ and $B_{\mathrm{D}}^{\prime \prime}$ were calculated. The $3 \times 2$ mixed ANOVA (stimulus type $\times$ set size) conducted on the hit data indicated only one reliable effect, the interaction between stimulus type and set size $\left[F(2,33)=4.82, M S_{\mathrm{e}}=0.0076\right.$, $p<.05, \eta^{2}=.23$; see Table 3]. Subsequent simple main effect (SME) analyses indicated the difference between memory sets to be dependent on the stimulus type. With matrices, performance was reliably better with memory sets of four items than with those of five items $[F(1,33)=6.17$, $\left.M S_{\mathrm{e}}=0.011, p<.05, \eta^{2}=.36\right]$. Visually similar inverted faces exhibited a similar trend, but this failed to be reliable $\left[F(1,33)=2.22, M S_{\mathrm{e}}=0.016, p>.05, \eta^{2}=.17\right]$, whereas with visually dissimilar inverted faces, the advantage for four-item lists over five-item lists was minimal $[F(1,33)=$ $\left.0.16, M S_{\mathrm{e}}=0.005, p>.05, \eta^{2}=.01\right]$. 
Analysis of the $A^{\prime}$ values revealed an effect of set size $\left[F(1,33)=14.14, M S_{\mathrm{e}}=0.003, p<.001, \eta^{2}=.30\right]$, indicating better discrimination with four-item than with fiveitem lists and a reliable set size $\times$ stimulus type interaction $\left[F(2,33)=6.90, M S_{\mathrm{e}}=0.003, p<.05, \eta^{2}=.30\right]$. Consistent with the hit data, SME analyses revealed better performance with four-item lists than with five-item lists only for matrices $\left[F(1,11)=17.63, M S_{\mathrm{e}}=0.004, p<.01, \eta^{2}=.62\right]$. Although visually similar inverted faces exhibited a similar difference, this failed to be reliable $\left[F(1,11)=3.04, M S_{\mathrm{e}}=\right.$ $0.001, p>.05]$, whereas with visually dissimilar inverted faces, the difference between four- and five-item lists was minimal $\left[F(1,11)=0.018, M S_{\mathrm{e}}=0.002, p>.05\right]$. Finally, analyses of the $B_{\mathrm{D}}^{\prime \prime}$ values revealed no reliable effects.

The serial position data were consistent with those from Experiment 2. The reliable position effect with four memory items $\left[F(3,99)=34.05, M S_{\mathrm{e}}=0.029, p<.001, \eta^{2}=\right.$ $.51]$ was the result of the last item's being more accurately identified than were any of the previous items, which did not differ. This pattern was also observed with memory set size five $\left[F(4,132)=23.28, M S_{\mathrm{e}}=0.035, p<.001, \eta^{2}=\right.$ .41 ; see Figure 5]. Some evidence of an extended recency effect was observed, with the Bonferroni tests indicating an advantage for Position 4 over Position 2.

Model fitting. The $c$ parameters resulting from fitting the SIMPLE model to the individual-participant data were subjected to a $2 \times 2$ mixed ANOVA (stimulus type $\times$ set size). This indicated that $c$ values derived from four-item lists were reliably higher than those from five-item lists $\left[F(1,33)=4.52, M S_{\mathrm{e}}=1.14, p<.05, \eta^{2}=.12\right.$; see Table 3]. This was accompanied by a stimulus $\times$ set size interaction $\left[F(2,33)=2.53, M S_{\mathrm{e}}=1.14, p>.05\right]$, which mirrored the effects observed in the hit and the $A^{\prime}$ data, indicating that the difference between the set sizes was reliable for matrices $\left[F(1,11)=6.65, M S_{\mathrm{e}}=1.19, p<.05, \eta^{2}=\right.$ .14], but not for visually similar inverted faces $[F(1,11)=$ $\left.1.07, M S_{\mathrm{e}}=1.71, p>.05\right]$ or for visually dissimilar inverted faces $\left[F(1,11)=0.10, M S_{\mathrm{e}}=0.05, p>.05\right]$.

The number of items contributing proactive interference was again estimated by monitoring the proportional change in RMSE. As in the two previous experiments, the best-fit functions, with one exception, resulted from allowing current list items and those from one preceding list to produce interference effects (see Table 4). The exception was with memory lists of five inverted faces, where the change in RMSE fell below 5\% only when interference was generated by two preceding trials.

\section{Discussion}

Reversing the order in which the memory sets were encountered did reverse the relationship between the $c$ values for the different memory list lengths. In Experiment 2, lists with five items were always encountered last, producing functions with higher $c$ values. When five-item lists were presented first, these produced lower $c$ values than did fouritem lists. Such a reversal supports the view that the visual distinctiveness of unfamiliar stimulus classes varies within the duration of the experiment, with items becoming more distinctive as familiarity with the stimulus class increases. This is further supported by the observation of the stimulus type $\times$ list length interactions observed for hits, $A^{\prime}$ and $c$ values, which together indicate that this effect is greatest for the stimulus type least encountered-namely, matrices. Although inverted faces are infrequently encountered, they are still recognized as faces and may be capable of utilizing some of the encoding mechanisms employed by faces (Murray, 2004), suggesting that strategies for discriminating exemplars may be acquired more quickly.

As in the previous experiments, the data were well fit by SIMPLE and confirm the relationship linking low $c$ values with reduced recency. Matrices again produced the lowest $c$ values and functions with only last-item recency. In contrast, inverted faces had higher $c$ values and demonstrated increased recency, especially with five-item memory lists. These data were also consistent in suggesting that in this STVM task, interference from items from the previous list are involved and responsible for the reduced primacy effect, confirming that the low levels of primacy are the result of local interference from more than the current list.

\section{GENERAL DISCUSSION}

The primary objective of these experiments was to investigate the adequacy of SIMPLE in explaining and predicting probe task performance when applied to STVM. In all three experiments, the empirical data were well fit by a version of the SIMPLE with one free parameter. Not only did this version of the model produce excellent fits to the empirical data from all three experiments, it also made two predictions that were upheld. The first relates to the magnitude of the recency effect. The proposal that serial position functions obtained with visual material exhibit only last-item recency (e.g., Avons, 1980, 1998; Broadbent \& Broadbent, 1981; Hanna \& Loftus, 1993; Kerr et al., 1999; Korsnes et al., 1996; Walker et al., 1993) is clearly not supported. Our results demonstrate that the amount of recency is crucially determined by the size of the $c$ parameter. SIMPLE predicts that an extended recency effect will emerge when $c$ values are high, which was observed in Experiment 1 with faces, and reduced recency when $c$ values are lower, as in Experiments 2 and 3 with inverted faces and random matrices.

The second prediction relates to the magnitude of the primacy effect. The empirical data from all the experiments reported here consistently produce functions with no measurable primacy. More important, these data are better fit by functions in which the interference locality extends outside the current trial items and are consistent with the simulation data in indicating that the best fits are obtained when interference neighborhood includes the items from just one previous trial.

Together, these findings elaborate one of the key assumptions upon which SIMPLE is built-namely, the local distinctiveness principle. This states that "the distinctiveness of an item in a memory task is dependent on the psychological distance from its nearest neighbours rather than on its distance from every member of the list of items to be discriminated" (Brown et al., 2002, p. 77). Embedded in this principle are the two key concepts of neighborhood size and psychological distance. The findings from our experiments indicate that under conditions 
in which the memory lists contain small numbers of items and successive trials are temporally near, the interference locality extends to include items from previous lists.

Our results also offer insights into the factors that influence the magnitude of the index of psychological distinctiveness within the visual domain. In SIMPLE, an item is distinctive and, therefore, better recognized when it is located in a sparsely populated region of psychological space. Time is obviously a key dimension in this multidimensional space, giving rise to the serial position functions obtained here. In Experiments 1 and 2, our attempts to identify other salient dimensions of this space involved attempting to produce changes in $c$, the index of psychological distinctiveness. In Experiment 1, this involved varying the visual similarity of upright faces. However, this manipulation failed to
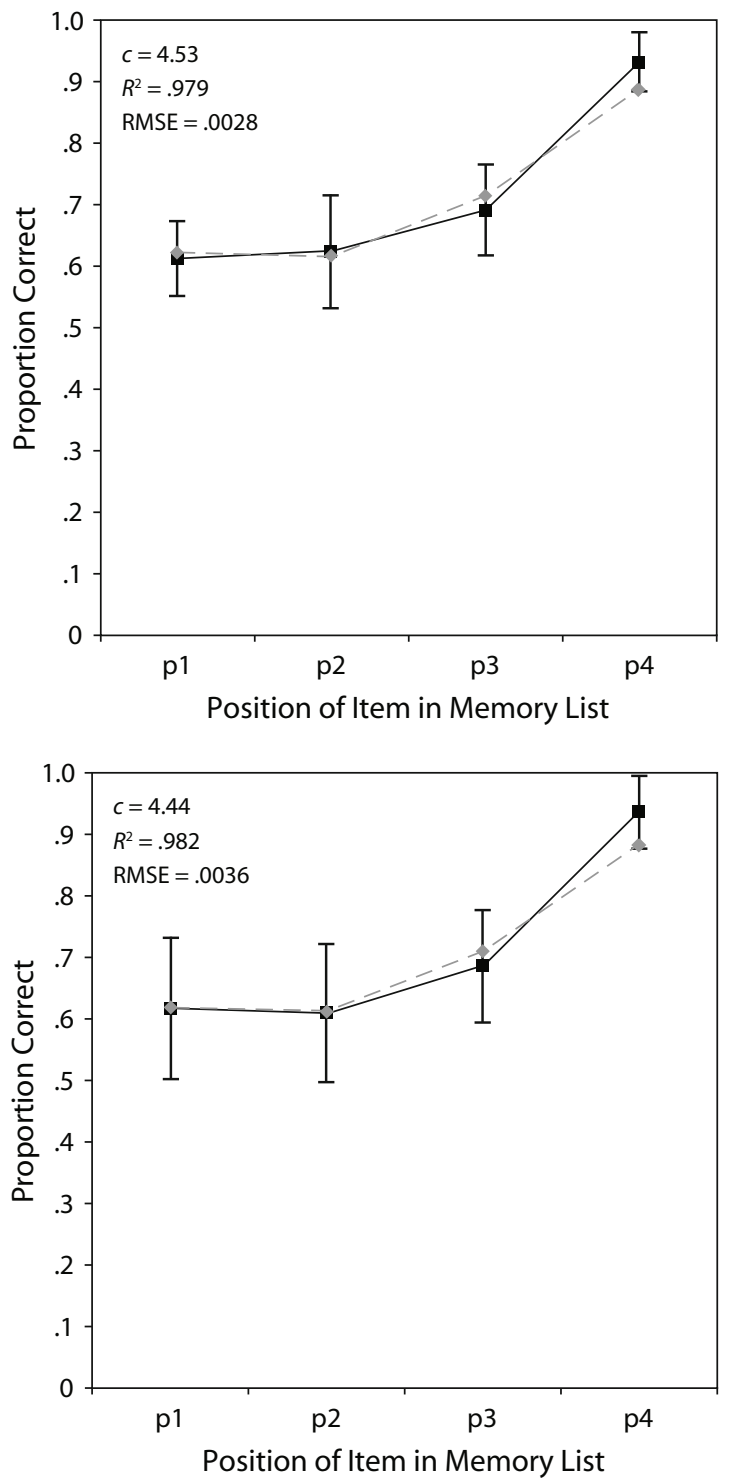

produce measurable changes in $c$, which Experiments 2 and 3 revealed to be associated only with changes in stimulus class familiarity. More specifically, psychological distinctiveness was found to be associated with both the population density of the representational space at the start of the experiment and large increases in density, relative to preexperimental density, that accrue during the course of the experiment. Both the preexperimental population density and the changes in density that accrue within an experiment are the result of perceptual learning and have been shown to be accompanied by increases in both memory and perceptual sensitivity (Palmeri, Wong, \& Gauthier, 2004).

Of the stimulus classes employed here, upright faces are the most frequently encountered. Valentine (1991) has proposed a scheme in which faces are represented in
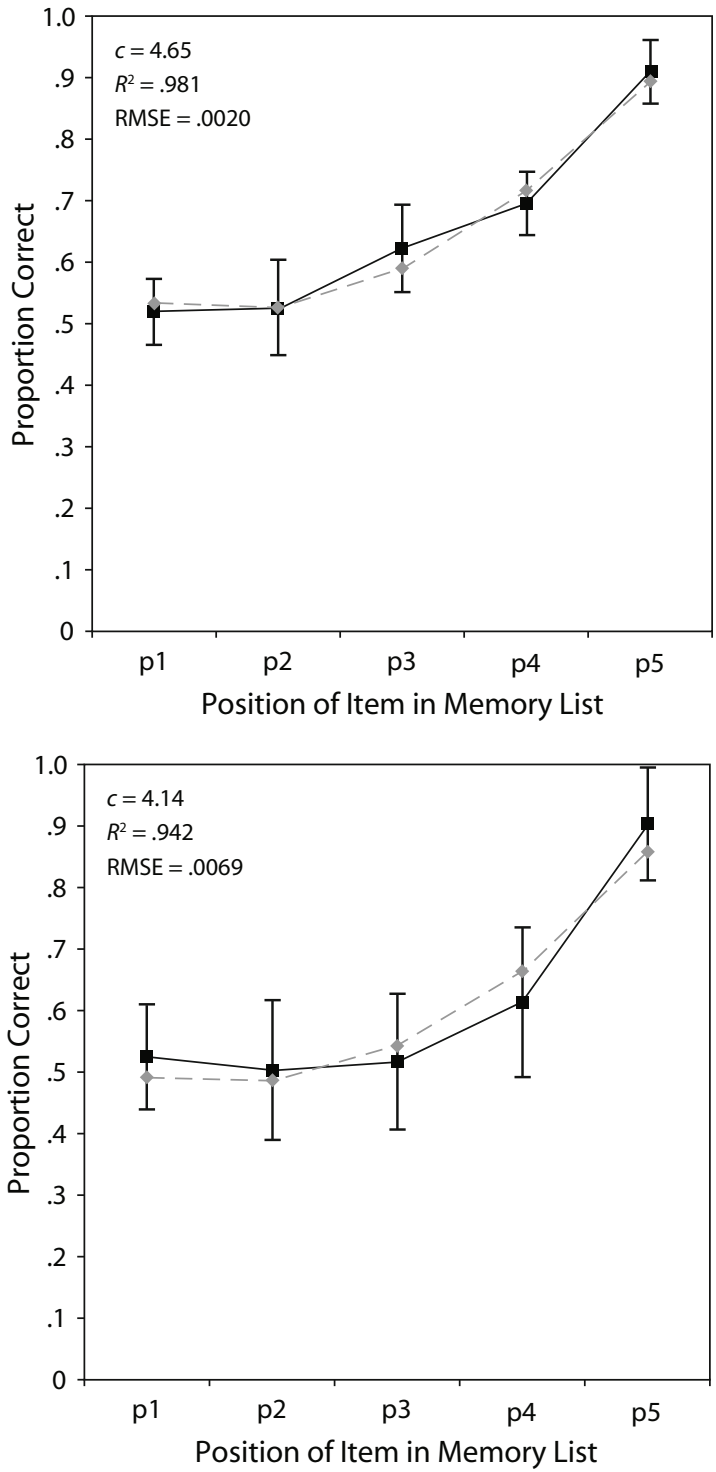

Figure 5. The proportion of inverted faces (upper panels) and matrices (lower panels) correctly identified as being previously seen, as a function serial position in Experiment 3. Data when four memory items were presented are shown in the left panel, and those when five memory items were presented are shown in the right panel. In this experiment, memory lists with five items always preceded lists with four items. 
Table 4

Values of the Power Index $c$ From the Best-Fitting Serial Position Functions for Four and Five Memory Items Generated by Fitting the SIMPLE Model to the Data From Experiment 3 (Inverted Faces and Random Matrices)

\begin{tabular}{|c|c|c|c|c|c|c|c|c|c|c|}
\hline \multirow[b]{2}{*}{ Stimulus } & \multicolumn{5}{|c|}{ Memory List Length 4} & \multicolumn{5}{|c|}{ Memory List Length 5} \\
\hline & $\begin{array}{l}\text { No. } \\
\text { Items }\end{array}$ & $c$ & $R^{2}$ & RMSE & $\begin{array}{l}\text { Prop. } \\
\text { Change }\end{array}$ & $\begin{array}{l}\text { No. } \\
\text { Items }\end{array}$ & $c$ & $R^{2}$ & RMSE & $\begin{array}{l}\text { Prop. } \\
\text { Change }\end{array}$ \\
\hline \multirow[t]{4}{*}{ Inverted faces } & 4 & 4.15 & .890 & .01105 & & 5 & 4.27 & .865 & .01533 & \\
\hline & 8 & 4.53 & .980 & .00279 & .748 & 10 & 4.63 & .980 & .00221 & .856 \\
\hline & 12 & 4.56 & .978 & .00266 & .047 & 15 & 4.65 & .981 & .00199 & .099 \\
\hline & 16 & 4.56 & .978 & .00265 & .004 & 20 & 4.65 & .982 & .00197 & .010 \\
\hline \multirow[t]{4}{*}{ Random matrices } & 4 & 4.07 & .922 & .01415 & & 5 & 3.72 & .923 & .01385 & \\
\hline & 8 & 4.44 & .982 & .00356 & .688 & 10 & 4.13 & .941 & .00706 & .490 \\
\hline & 12 & 4.47 & .978 & .00348 & .022 & 15 & 4.17 & .936 & .00752 & -.065 \\
\hline & 16 & 4.48 & .978 & .00347 & .003 & 20 & 4.18 & .935 & .00765 & -.008 \\
\hline
\end{tabular}

Note-For each function, two indices of fit between observed and predicted data are presented: the squared correlation $\left(R^{2}\right)$ and the root-mean square error (RMSE). For each stimulus type, fits are provided for differing numbers of items allowed to produce interference effects, along with the proportional change in RMSE associated with each increase.

memory as points in a multidimensional space, with dimensions developed to best discriminate exemplars. In a series of experiments, he has shown how his face space model can account for the effects of distinctiveness, inversion, and race in face recognition (Valentine, 1991; Valentine \& Endo, 1992). Because of the greater exposure to upright faces, this face space is densely populated with a well-defined structure developed to maximally distinguish faces. This view is consistent with image-based models of object recognition, which show greater representational discriminability as the density of the stored views within a representational space increases (Edelman, 1999; Riesenhuber \& Poggio, 1999). Familiarity with the stimulus material not only increases the distinctiveness of the memory representations, but also makes encoding more efficient. One such mechanism that is particularly important here is that of unitization (Goldstone, 1998), which involves the construction of functional units that are responsive to complex configurations. This is evident in the developmental change from featural to configurational processing observed in children's face-processing abilities, which is disrupted by inversion (Diamond \& Carey, 1986; Hay \& Cox, 2002). Thus, employing faces in Experiment 1 invoked the use of extremely efficient and specialized encoding and storage systems that are reflected in the high values of the $c$ observed and the small changes in this parameter related to the visual similarity manipulations employed here.

In contrast, inverted faces, random matrices, and the other forms of abstract visual stimuli are unlikely to have ever been previously experienced. Whereas the population density and the structure of the representational space for faces are relatively unaffected by the number of additional face exemplars presented in these experiments, this is not the case for inverted faces and random matrices. The representational spaces of both classes are essentially empty and become increasingly more populated during the course of the experiment. In Experiment 2, the effect of this perceptual learning was to improve performance on the last block of trials. Since this block always contained five memory items, this resulted in removing the performance advantage normally associated with smaller memory lists. In Experiment 3 , reversing the list orders again led to a performance improvement with the last block. Not only did this produce the expected performance advantage for four-item lists, but also the results indicate that the perceptual learning was dependent on the stimulus type. We suggest that the observance of different learning rates for the different stimulus classes in Experiment 3 is linked to the exposure to greater numbers of exemplars in the first block. Since the block of five-item memory lists contained more trials, this led to a greater representational density and, as a result, allowed more perceptual learning to take place than in Experiment 2 , in which the block of four-item lists had fewer trials. In addition, the observation of a reliably greater effect with matrices than with inverted faces is consistent with the proposal made by McLaren, Leevers, and Mackintosh (1994). Using checkerboard stimuli, they found faster perceptual learning with stimuli derived from a prototype. In the present study, perceptual learning was faster with inverted faces, which are prototype based, producing performance improvements within the first trial block and, thus, reducing differences across block for this stimulus class.

It is interesting to note that Kahana and Sekuler (2002) also reported an extended recency effect when using textures created by varying vertical and horizontal special frequencies. These are an unfamiliar, abstract stimulus class predicted to have low psychological distinctiveness and, consequently, expected to have flat functions with last-item recency. However, in this study, there were only 27 memory items, and each participant completed 1,800 trials across five sessions. The small population of exemplars, coupled with the large number of repeated exposures, was sufficient for the perceptual-learning factors outlined above to have played a major role. If this is the case, this suggests that the psychological distinctiveness of the items increased during the course of the experiment, culminating in levels similar to those for faces and exhibiting functions with extended recency. Unfortunately, the primary interest in Kahana and Sekuler's study was pattern recognition, and not perceptual learning, so any changes in function shape remain unknown. 
In summary, we have shown that SIMPLE is good at both predicting and describing the changes in STVM probe functions that result from varying stimulus properties. More important, we have provided an explanation for the variety of function shapes that can occur and have also shown the importance of changes that result from perceptual learning when unfamiliar, abstract stimulus classes are employed. An important task for future research is to map the scope of the changes in psychological distinctiveness that occur with increasing exposure to items from different stimulus classes and to relate these to changes in function shape.

\section{AUTHOR NOTE}

Correspondence regarding this article should be addressed to D. C. Hay, Department of Psychology, Fylde College, Lancaster University, Bailrigg, Lancaster LA1 4YF, England (e-mail: d.hay@lancaster .ac.uk).

\section{REFERENCES}

Ashby, F. G., \& Townsend, J. T. (1986). Varieties of perceptual independence. Psychological Review, 93, 154-179.

Avons, S. E. (1980). Visualization and memorization. Unpublished doctoral dissertation, University of Stirling.

Avons, S. E. (1998). Serial report and item recognition of novel visual patterns. British Journal of Psychology, 89, 285-308.

Avons, S. E., Wright, K. L., \& PAMmer, K. (1994). The word-length effect in probed and serial recall. Quarterly Journal of Experimental Psychology, 47A, 207-231.

Broadbent, D. E., \& Broadbent, M. H. P. (1981). Recency effects in visual memory. Quarterly Journal of Experimental Psychology, 33A, 1-15.

Brown, G. D. A., Neath, I., \& Chater, N. (2002). A ratio model of scale-invariant memory and identification (Memory Lab Tech. Rep. No. 2002-01). Purdue University.

Bruce, V., \& Young, A. W. (1986). Understanding face recognition. British Journal of Psychology, 73, 105-116.

DiAMOND, R., \& CAREY, S. (1986). Why faces are and are not special: An effect of expertise. Journal of Experimental Psychology: General, 115, 107-117.

EDELMAN, S. (1999). Representation and recognition in vision. Cambridge, MA: MIT Press.

Ellis, H. D. (1975). Recognising faces. British Journal of Psychology, 66, 409-426.

GoldSTONE, R. L. (1998). Perceptual learning. Annual Review of Psychology, 49, 585-612.

HANNA, A., \& LofTUS, G. (1993). A model for conceptual processing of naturalistic scenes. Canadian Journal of Experimental Psychology, 47, 548-569.

HAY, D. C., \& Cox, R. (2002). Developmental changes in the recognition of faces and facial features. Infant \& Child Development, 9, 199-212.

HaY, D. C., \& Young, A. W. (1982). The human face. In A. W. Ellis (Ed.), Normality and pathology in cognitive function (pp. 173-202). London: Academic Press.

Hunt, R. R. (2003). Two contributions of distinctive processing to accurate memory. Journal of Memory \& Language, 48, 811-825.

Kahana, M. J., \& SeKuler, R. (2002). Recognising spatial patterns: A noisy exemplar approach. Vision Research, 42, 2177-2194.

KERR, J. R., AvONS, S. E., \& WARD, G. (1999). The effect of retention interval on serial position curves for item recognition of visual patterns and faces. Journal of Experimental Psychology: Learning, Memory, \& Cognition, 25, 1475-1494.

KoRSNES, M. S., MaGnussen, S., \& Reinvang, I. (1996). Serial position effects in visual short-term memory for words and abstract spatial patterns. Scandinavian Journal of Psychology, 37, 62-73.

Leder, H., Candrian, G., Huber, O., \& Bruce, V. (2001). Configural features in the context of upright and inverted faces. Perception, 30, 73-83.

MANNING, S. K., \& SCHREIER, H. (1988). Recency and suffix effects in pictures as a function of recall method. American Journal of Psychology, 101, 97-109.

McLaren, I. P. L., Leevers, H. L., \& Mackintosh, N. J. (1994). Recognition, categorization and perceptual learning (or, how learning to classify things together helps one to tell them apart). In C. Umiltà \& M. Moscovitch (Eds.), Attention and performance XV: Conscious and nonconcious information processing (pp. 889-909). Cambridge, MA: MIT Press, Bradford Books.

Moses, Y., Ullman, S., \& Edelman, S. (1996). Generalisation to novel images in upright and inverted faces. Perception, 25, 443-461.

Murdock, B. B., JR. (1960). The distinctiveness of stimuli. Psychological Review, 67, 16-31.

MURRAY, J. E. (2004). The ups and downs of face perception: Evidence for holistic encoding of upright and inverted faces. Perception, 33, 387-398.

MurRay, J. E., Yong, E., \& Rhodes, G. (2000). Revisiting the perception of upside-down faces. Psychological Science, 11, 492-496.

Nairne, J. S., Riegler, G. L., \& SerRA, M. (1991). Dissociative effects of generation on item and order retention. Journal of Experimental Psychology: Learning, Memory, \& Cognition, 17, 702-709.

NAIRNe, J. S., Whiteman, H. L., \& WoesSNER, L. (1995). Symmetrical cuing effects for item and position information. American Journal of Psychology, 108, 345-358.

NEATH, I. (1993). Distinctiveness and serial position effects in recognition. Memory \& Cognition, 21, 689-698.

NeAth, I., \& BROWN, G. D. A. (2006). SIMPLE: Further applications of a local distinctiveness model of memory. In B. H. Ross (Ed.), Psychology of learning and motivation (Vol. 46, pp. 210-243). San Diego: Academic Press.

Nosofsky, R. M. (1986). Attention, similarity and the identificationcategorization relationship. Journal of Experimental Psychology: General, 115, 39-57.

PaLMERI, T. J., Wong, A. C. N., \& GAuthiER, I. (2004). Computational approaches to the development of perceptual expertise. Trends in Cognitive Sciences, 8, 378-386.

Phillips, W. A., \& Christie, D. F. M. (1977). Components of visual memory. Quarterly Journal of Experimental Psychology, 29, 117-133.

Pollack, I., \& Norman, D. A. (1964). A non-parametric analysis of recognition experiments. Psychonomic Science, 1, 125-126.

Riesenhuber, M., \& Poggio, T. (1999). Hierarchical models of object recognition in cortex. Nature Neuroscience, 2, 1019-1025.

Smyth, M. M., Hay, D. C., Hitch, G. J., \& Horton, N. J. (2005). Serial position memory in the visual-spatial domain: Reconstructing sequences of unfamiliar faces. Quarterly Journal of Experimental Psychology, 58A, 909-930.

Sternberg, S. (1966). High-speed scanning in human memory. Science, 153, 652-654.

VALENTINE, T. (1991). A unified account of the effects of distinctiveness, inversion, and race in face recognition. Quarterly Journal of Experimental Psychology, 43A, 161-204.

VALENTine, T. (ED.) (1995). Cognitive and computational aspects of face recognition: Explorations in face space. London: Routledge.

VAlentine, T., \& Endo, M. (1992). Towards an exemplar model of face processing: The effects of race and distinctiveness. Quarterly Journal of Experimental Psychology, 44A, 671-703.

Walker, P., Hitch, G. J., \& Duroe, S. (1993). The effect of visual similarity on short-term memory for spatial location: Implications for the capacity of visual short-term memory. Acta Psychologica, 83, 203-224.

WARD, G., Avons, S. E., \& Melling, L. (2005). Serial position curves in short-term memory: Functional equivalence across modalities. Memory, 13, 308-317.

(Manuscript received November 3, 2004; revision accepted for publication November 16, 2005.) 